\title{
Exact Mean-Field Hamiltonian for Fermionic Lattice Models in High Dimensions
}

\author{
P. G. J. van Dongen ${ }^{(a)}$ and D. Vollhardt \\ Institut für Theoretische Physik C, Technische Hochschule Aachen, \\ D-5100 Aachen, Federal Republic of Germany
}

(Received 14 May 1990)

\begin{abstract}
We show that even for fermionic lattice models with on-site interaction a mean-field Hamiltonian can be constructed, which - in analogy with spin lattice models- becomes exact in the limit of high spatial dimensions $d$. Here the mean fields are fermion operators, rather than numbers. We use this method to obtain the exact solution of a simplified Hubbard model on a Bethe lattice for all temperatures. The order parameter is found to have the conventional mean-field form, but exhibits unusually rich behavior as a function of the interaction.

PACS numbers: $75.10 . \mathrm{Jm}, 71.10 .+\mathrm{x}, 75.10 . \mathrm{Lp}$
\end{abstract}

Mean-field theories (MFTs) play an important role in classical and quantum-mechanical many-body problems. Although they are based on a gross simplification of the underlying physics they often provide a good qualitative description of the properties of a system. To assess the validity of a MFT one needs to know whether it becomes exact in some limit, such that it can be improved systematically, e.g., by perturbation theory around this limit. It is well established that for classical and quantum spin lattice models (e.g., Ising, Heisenberg) the meanfield expressions for the free energies become exact for high coordination number $Z$, i.e., for spatial dimension $d \rightarrow \infty .^{1,2}$ For example, in the MFT for the Ising model the local field at a given site is replaced by its expectation value, which is entirely described by a single variable, namely, the order parameter (here, the magnetization). It is intuitively clear that this approximation becomes exact for $d \rightarrow \infty$, since a given spin "sees" a local field composed of $Z \sim O(d)$ neighboring spins, whose fluctuations vanish in the limit $d \rightarrow \infty$.

The situation is fundamentally different in the case of fermionic lattice models, i.e., models with itinerant quantum-mechanical degrees of freedom. Typical examples are the Hubbard model ${ }^{3}$ and related models for interacting lattice electrons, which are employed in the study of itinerant magnetism, the metal-insulator transition, and high- $T_{c}$ superconductivity. For nearestneighbor hopping a generalized Hubbard model has the form

$$
\begin{aligned}
& H=H_{\mathrm{k} \text { In }}+H_{\mu}+H_{U}+H_{V}, \\
& H_{\mathrm{kin}}=\sum_{\langle\mathrm{i}\rangle\rangle, \sigma} t_{\sigma} \hat{c}_{\mathrm{i} \sigma}^{\dagger} \hat{c}_{\mathrm{j} \sigma}, \quad H_{\mu}=-\sum_{\mathrm{i} \sigma} \mu_{\sigma} \hat{n}_{\mathrm{i} \sigma}, \\
& H_{U}=U \sum_{\mathrm{i}} \hat{n}_{\mathrm{i} \uparrow} \hat{n}_{\mathrm{i} \downarrow}, \quad H_{V}=\sum_{\langle\mathrm{ij}\rangle} \sum_{\sigma \sigma^{\prime}} V_{\sigma \sigma^{\prime}} \hat{n}_{\mathrm{i} \sigma} \hat{n}_{\mathrm{j} \sigma^{\prime}},
\end{aligned}
$$

where $\hat{c}_{\mathbf{i} \sigma}^{\dagger}\left(\hat{c}_{\mathrm{i} \sigma}\right)$ creates (destroys) a $\sigma$ spin at site $\mathbf{i}$, with $\hat{n}_{\mathbf{i} \sigma}=\hat{c}_{\mathbf{i} \sigma}^{\dagger} \hat{c}_{\mathbf{i} \sigma}$. In the original Hubbard model ${ }^{3}$ the hopping constant $t_{\sigma}$ is independent of spin, and the interaction is purely on site. A further simplification can be introduced if only one of the two spin species is allowed to hop $\left(t_{\downarrow}=-t, t_{\uparrow}=0\right)$. This simplified Hubbard model was discussed as an approximation of the full model. ${ }^{4}$ It was also considered by Falicov and Kimball ${ }^{5}$ as a model for a semiconductor-metal transition and by Kennedy and Lieb $^{6}$ as a model for crystallization. In this model the $\uparrow$ spins have been proved to form a checkerboard pattern at sufficiently low temperatures and for half filling, at least on $A B$ lattices. ${ }^{6,7}$

The conventional MFT for (1) is Hartree-Fock theory, where the interaction is factorized; i.e., spatial fluctuations are neglected as in the MFT for spin lattice models. The question then arises whether here, too, this approximation becomes exact in $d=\infty$. The limit of high dimensions for Hubbard-type models has recently been introduced by Metzner and Vollhardt. ${ }^{8}$ It has since been shown to be very useful in a number of problems, ${ }^{9-12}$ since many essential features of systems in $d=3$ (and even lower) dimensions are well described by the results in $d=\infty$. Making use of the fact that in $d=\infty$ the proper self-energy becomes site diagonal, ${ }^{8,9}$ the simplified Hubbard model was solved exactly in $d=\infty$ by Brandt and Mielsch, ${ }^{11}$ who showed that it is sufficient to solve the atomic problem in a generalized, time-dependent external field. From the diagrammatic expression for the ground-state (or the self-) energy it follows that for the Hubbard model Hartree-Fock theory does not become exact in $d=\infty .8,9.13$ Indeed, fluctuations in the on-site interaction, which are neglected within Hartree-Fock theory, never become small, since

$$
\left\langle\left(\hat{n}_{\mathbf{i} \uparrow}-\left\langle\hat{n}_{\mathbf{i} \uparrow}\right\rangle\right)\left(\hat{n}_{\mathbf{i} \downarrow}-\left\langle\hat{n}_{\mathbf{i} \downarrow}\right\rangle\right)\right\rangle \sim O(1),
$$

independent of dimension. Therefore, of all interactions in (1) it is precisely the on-site interaction that remains as the only dynamical interaction in $d=\infty$. ${ }^{9}$ This conclusion equally holds for the simplified Hubbard model, at least for fixed $U>0$ and temperature $T>0$.

We will now show how to construct a mean-field Hamiltonian for Hubbard-type models which does become exact in $d=\infty$. We will mainly consider the simplified Hubbard model $H^{\text {simp }}$, i.e., $t_{\uparrow}=0, t_{\text {. }}$ $=-\bar{t} / Z^{1 / 2}$ with fixed $\bar{t}$ (in the following $\left.\bar{t} \equiv 1\right),{ }^{8}$ and $V_{\sigma \sigma^{\prime}}=0$ in (1). From the analysis of Ref. 6 we conclude 
that it is the $A B$ structure of the lattice that determines the phase diagram. Therefore we will use a Bethe lattice with coordination number $Z$, since in this case all calculations remain rather simple. (We will later see that for $Z \rightarrow \infty$ the results are indeed qualitatively the same as those for a $d=\infty$ hypercubic lattice.) Thereby we will not only recover the existing exact solution of the model in $d=\infty$ (Ref. 11) in a rather simple way, but will extend it into the ordered state and understand in which sense high dimensionality leads to exact mean-field features even in fermionic lattice systems.

To obtain a mean-field description we consider a site $\mathbf{i}$ and investigate the influence of the surrounding sites. The latter effect is described by the Green function $G_{\mathrm{ij}}\left(\tau-\tau^{\prime}\right)=-\left\langle T_{\tau} \hat{c}_{\mathrm{i} !}(\tau) \hat{c}_{\mathrm{j} !}^{\dagger}\left(\tau^{\prime}\right)\right\rangle$, whose time evolution is given by the equations of motion. In particular, for $\mathbf{i}=\mathbf{j}$ the first derivative $\partial G_{\mathrm{ii}} / \partial \tau$ involves the commutator [ $\left.H^{\text {simp }}, \hat{c}_{\mathrm{i} \downarrow}\right]$, the second derivative $\left[H^{\text {simp }},\left[H^{\text {simp }}, \hat{c}_{\mathrm{i} \downarrow}\right]\right]$, etc. In the limit $Z \rightarrow \infty$ these commutators depend only on a few dynamical degrees of freedom. For instance, [ $\left.H^{\text {simp }}, \hat{c}_{\mathrm{i} !}\right]$ introduces only two new fermions $\hat{c}_{e}$ and $\hat{c}_{f}$ which represent the entire first shell of neighbors of site i:

$$
\begin{aligned}
& \hat{c}_{e} \equiv\left[\left(1-\rho_{1}\right) Z\right]^{-1 / 2} \sum_{|\mathbf{i}-\mathbf{j}|=1}\left(1-\hat{n}_{\mathrm{j} \mid}\right) \hat{c}_{\mathbf{j} !}, \\
& \hat{c}_{f} \equiv\left(\rho_{1} Z\right)^{-1 / 2} \sum_{|\mathrm{i}-\mathrm{j}|=1} \hat{n}_{\mathbf{j} \uparrow} \hat{c}_{\mathrm{j} !} .
\end{aligned}
$$

The fermion operators $\hat{c}_{f}$ ("filled") and $\hat{c}_{e}$ ("empty") describe annihilation of a $\downarrow$ spin in the first shell provided there is, or is not, an $\uparrow$ spin present. The high dimensionality becomes manifest in the prefactors in $(2 \mathrm{a})$ and (2b): Only for $Z \rightarrow \infty$ can one express the normalization constants of the few fermions in terms of the density $\rho_{l}$ of $\uparrow$ spins in the $l$ th shell (here $l=1$ ). Similarly, the calculation of $\partial^{2} G_{\mathrm{ii}} / \partial \tau^{2}$ yields four new fermions: $\hat{c}_{e e}$, $\hat{c}_{e f}, \hat{c}_{f e}$, and $\hat{c}_{f f}$, representing all possible paths from site $i$ to the second shell, where a $\downarrow$ spin is annihilated. In general, the $n$th shell enters only via the $n$th derivative of $G_{\mathrm{ii}}$ and is described by $2^{n}$ fermions $\hat{c}_{\mathrm{X}}$, representing the possible ways $\mathbf{X}=\left(x_{1}, \ldots, x_{n}\right)$, with $x_{l}=e, f$, to travel from site $\mathrm{i}$ to the $n$th shell (see Fig. 1).

The essential point is that the commutators occurring in $\partial^{n} G_{\mathrm{ii}} / \partial \tau^{n}$ may equally be derived from an effective Hamiltonian $H^{\mathrm{MF}}$ that has the same form as $H^{\text {simp }}$ but where $H_{t}^{\mathrm{MF}}, H_{U}^{\mathrm{MF}}$, and $H_{\mu}^{\mathrm{MF}}$ only depend on the collective variables $\hat{c} \mathbf{X}$, namely,

$$
\begin{aligned}
& H_{t}^{\mathrm{MF}}=-\sum_{l(\mathbf{X}) \geq 0}\left[\left(1-\rho_{l+1}\right)^{1 / 2} \hat{c}_{\mathbf{X} e}^{+} \hat{c} \mathbf{X}\right. \\
& +\rho_{l+1}^{l / 2} \hat{c}_{\mathbf{X}}^{+} \hat{c}_{\mathbf{X}}+\text { H.c.] } \\
& H_{\mu}^{\mathrm{MF}}=-\left(\mu_{!} \hat{n}_{\mathrm{i} \downarrow}+\mu_{\uparrow} \hat{n}_{\mathrm{i}\rceil}\right. \\
& \left.+\sum_{l(\mathbf{X}) \geq 0}\left[\mu_{l} \hat{n}_{\mathbf{X} e}+\left(\mu_{\downarrow}-U\right) \hat{n}_{\mathbf{X} f}\right]\right), \\
& H_{\ell^{\prime}}^{\mathrm{MF}}=U \hat{n}_{\mathbf{i} \uparrow} \hat{n}_{\mathrm{i} \downarrow},
\end{aligned}
$$

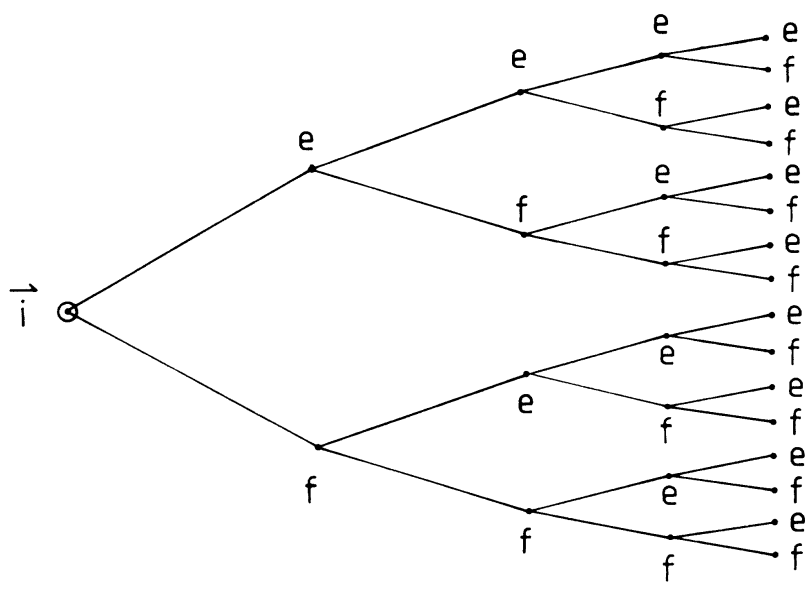

FIG. 1. Effective Bethe lattice on which the mean-field Hamiltonian $H^{\mathrm{MF}}$, Eq. (3), operates; here $f$ and $e$ denote the presence and absence of an $\uparrow$ spin.

where $l(X)$ is the length of the vector $\mathbf{X}$. The notation $\mathbf{X} e$ represents the $(l+1)$-dimensional vector $(\mathbf{X}, e)$, and similarly for $\mathbf{X} f$. Note that $\boldsymbol{H}^{\mathrm{MF}}$ has a dependence on site i, which, however, only distinguishes between the $A$ and $B$ sublattices. The fact that $H^{\text {simp }}$ and $H^{\mathrm{MF}}$ in (3) generate the same time evolution can readily be proved by induction. We conclude that for $Z \rightarrow \infty$ the dynamics of the simplified Hubbard model reduces to that of a noninteracting, tight-binding model on a Bethe lattice with coordination number $Z^{M F}=3$.

The form of $H^{\mathrm{MF}}$ is very illustrative. The interaction term $H_{U}^{\mathrm{MF}}$ is strictly local: A Hartree-Fock decoupling does not occur. The surrounding sites enter only in an average way, namely, by modification of the chemical potentials in $H_{\mu}^{\mathrm{MF}}$. The kinetic energy $H_{t}^{\mathrm{MF}}$ contains hopping rates depending on the densities $\rho_{l}$. Since $\rho_{l}$ determines the order parameter in this model (see below), we see that $H^{\mathrm{MF}}$ has genuine mean-field character, in the sense that it is described by a single parameter as in the case of spin lattice models. Furthermore, in our approach the "mean fields" are collective fermion operators, rather than numbers.

Next we investigate whether the resulting critical properties are mean-field-like. The model has an Isinglike phase transition at half filling in dimensions $d \geq 2 ;^{6,7}$ for $U \rightarrow \infty$ it is even unitarily equivalent to the Ising model. ${ }^{6}$ In high dimensions one therefore expects that the order parameter (i.e., the density difference of the $\uparrow$ spins on the $A$ and $B$ sublattices) behaves like the magnetization in the Ising model. To substantiate these expectations we focus on the half-filled case $\left(\mu_{\uparrow}=\mu_{!}=U / 2\right)$. The Green functions $G_{\mathrm{ij}}$ can be calculated ${ }^{14}$ by using the standard techniques for noninteracting tight-binding models on the Bethe lattice. ${ }^{15,16}$ The $A B$ structure of the low-temperature phase implies $\rho_{2 l-1} \equiv \rho_{A}$ and $\rho_{2 l} \equiv \rho_{B}$ for all $l=1,2, \ldots$, so that the order parameter follows as $\Delta=\left|\rho_{A}-\rho_{B}\right|$. With the 
definition $\rho^{ \pm}=\frac{1}{2}(1 \pm \Delta)$ we write $G_{\mathrm{ii}}=G-$ if $\rho_{B}=\rho^{-}$, and $G_{\mathrm{ii}}=G_{+}$if $\rho_{B}=\rho^{+}$. One finds that $G_{+}(z)$, where $z=i \omega_{n}$ and $\omega_{n}$ is the Matsubara frequency, is the root satisfying $G_{+} \sim z^{-1}$ for $|z| \rightarrow \infty$ of the cubic equation

$$
-G_{+}^{*}\left[\left(z-G_{+}\right)^{2}-\frac{1}{4} U^{2}\right]=z-\frac{1}{2} U \Delta-G_{+},
$$

and $G_{-}=-G_{+}^{*}$. The off-diagonal Green functions $G_{\mathrm{ij}}$, with $|\mathbf{i}-\mathbf{j}|=1$, can be expressed in terms of $G+$ and $G+{ }^{16}$ The kinetic energy per site is now given as

$$
e_{t}=-\frac{2}{\beta} \sum_{\omega_{n}>0}\left|G_{+}\left(i \omega_{n}\right)\right|^{2} .
$$

The potential energy can be expressed in terms of $a \equiv \operatorname{Re} G_{+}(z)$ and $b \equiv \operatorname{Im} G_{+}(z)$. With the definition

$$
\zeta^{ \pm}(z) \equiv \frac{U / 2 \pm a}{(\omega-b)^{2}+(U / 2 \pm a)^{2}}
$$

one finds for the potential energy per site

$$
e_{U}=U\left(\frac{1}{4}-\frac{1}{\beta} \sum_{\omega_{n}>0}\left(\rho^{+} \zeta^{-}+\rho^{-} \zeta^{+}\right)\right)
$$

The internal energy is given by $e(\beta, \Delta)=e_{t}+e_{U}$, and the free energy is obtained by integrating the relation $e=\partial(\beta f) / \partial \beta$ as

$$
\begin{aligned}
\beta f(\beta, \Delta)=-\ln 2+\rho^{+} \ln \rho^{+} & +\rho^{-} \ln \rho^{-} \\
& +\int_{0}^{\beta} d x e(x, \Delta) .
\end{aligned}
$$

In order to localize the phase transition, we expand $f(\beta, \Delta)$ in terms of $\Delta^{2}$, which leads to the usual Ginzburg-Landau equation. In our model the prefactor of the $\Delta^{2}$ term, $f_{2}(\beta)$, can be calculated explicitly. The criterion $f_{2}\left(\beta_{c}\right)=0$ is found to be equivalent to the $T_{c}$ formula of Ref. 11. ${ }^{14}$ For small and large values of $U$ the critical temperature $T_{c}(U)$ is found as

$$
k_{B} T_{c} \sim\left\{\begin{array}{l}
(2 U)^{-1} \quad(U \rightarrow \infty), \\
\left(U^{2} / 2 \pi\right) \ln \left(U^{-1}\right) \quad(U \rightarrow 0) .
\end{array}\right.
$$

The large- $U$ result ( $8 \mathrm{a})$ is the critical temperature of the equivalent Ising model ${ }^{6}$ with coupling constant $(2 U)^{-1}$. The small- $U$ result ( $8 \mathrm{~b})$ is new and can be compared to the known bounds on $T_{c}$ : Kennedy and Lieb $^{6}$ showed that $T_{c}$ is bounded from below by $U^{2} /|\ln U|$ as $U \rightarrow 0$, and conjectured $T_{c} \propto U^{2}$. Equation (8b) shows that in fact $T_{c} \propto U^{2}|\ln U|$

Next we consider the temperature dependence of the equilibrium order parameter $\Delta_{0}(T)$. Near $T_{c}$ it follows from the expansion of $f(\beta, \Delta)$ that $\Delta_{0}(T) \propto\left(T_{c}-T\right)^{1 / 2}$, while at low temperatures $1-\Delta_{0} \propto \exp \left[-2 e_{1}(\infty) / k_{B} T\right]$, where $e_{1}(\beta) \equiv-\partial e(\beta, 1) / \partial \Delta$. Hence $\Delta_{0}$ has indeed the expected mean-field form. The order parameter can also be calculated exactly in the two limits $U \rightarrow \infty$ and $U \rightarrow 0$. In the Ising limit $U \rightarrow \infty$, one obtains the Curie-Weiss result $\Delta=\tanh (\Delta / \theta)$, with $\theta=T / T_{c}(U)$, while the results in the limit $U \rightarrow 0$ are less trivial (see

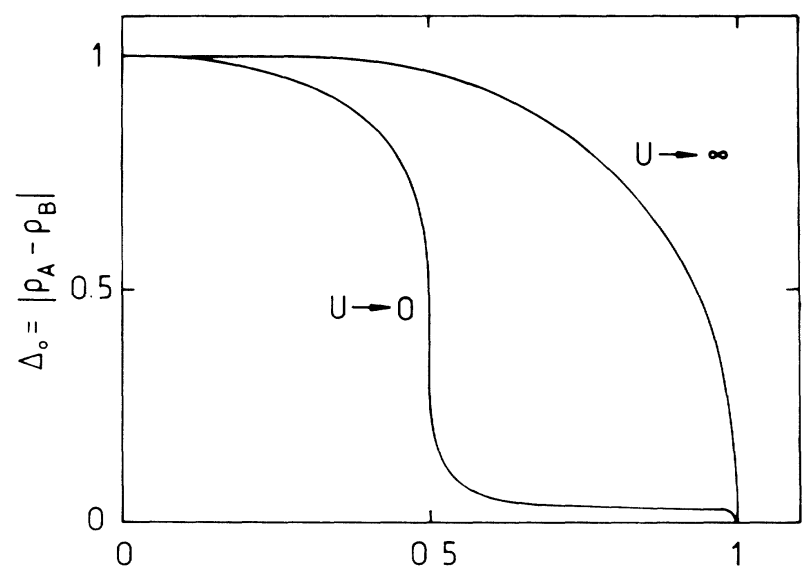

FIG. 2. The order parameter $\Delta_{0}(\theta, U)$, with $\theta=T / T_{c}(U)$, in the limits $U \rightarrow 0$ and $U \rightarrow \infty$.

Fig. 2). At $\theta_{c}=1, \Delta(\theta)$ has a square-root singularity, but the prefactor is extremely small (of order $U|\ln U|^{3 / 2}$ ). The order parameter remains small, proportional to $U^{2 \theta-1}$, for all $\theta>\frac{1}{2}$. Nonvanishing values of $\Delta$ for $U \rightarrow 0$ are found only for $0 \leq \theta<\frac{1}{2}$, where the result is remarkably simple: $\Delta=\tanh (\Delta / 2 \theta)$ for $U \rightarrow 0$. Thus there is an apparent critical temperature of $\frac{1}{2} T_{c}$. Somewhat surprisingly, one finds that these results for $U \rightarrow 0$ are identical to the corresponding Hartree-Fock predictions, ${ }^{14}$ which hence become exact in this limit.

The exact solution in $d=\infty$ can also be obtained in the presence of nearest-neighbor interactions, since this only leads to a modification of the chemical potentials. The phase diagram can be drastically different then. ${ }^{14}$ Our method can also be used on regular lattices, e.g., the hypercubic lattice. In this case there are $n$ ! possible paths of equal length $n$ between two lattice points $\mathbf{i}$ and $\mathbf{j}$ if $|\mathbf{i}-\mathbf{j}|=n$. Hence it does not suffice to consider fermions $\hat{c}_{\mathbf{X}}$ representing paths. Rather, one obtains a new fermion for every possible configuration on the hypercube formed by the union of all paths of length $n$ between $i$ and j. Nevertheless, the mean-field Hamiltonian still has the noninteracting tight-binding form, but this time on a lattice with a complicated topological structure involving loops. Apart from this the structure and interpretation of the mean-field Hamiltonian is identical. The extension of our method to the full Hubbard model is possible in principle. The hopping of the $\uparrow$ electrons destroys the structure of the $\hat{c}_{\mathbf{X}}$ operators, giving rise to many new fermions. However, in $d=\infty$ the Hubbard model and its simplified version coincide for large $U$ at half filling. Besides that our method directly yields the exact solution of the Hubbard model in simplified geometries, e.g., clusters. ${ }^{17}$

In summary, we have pointed out, and have clarified, the relation between high dimensions and mean-field theories for fermionic lattice systems. For the simplified Hubbard model on a Bethe lattice we showed that the 
exact solution for $Z \rightarrow \infty$ can be derived from a meanfield Hamiltonian which has a tight-binding form and is a simple function of the order parameter. ${ }^{18}$ This exact mean-field theory differs from Hartree-Fock theory (which generally does not become exact for $d \rightarrow \infty$ ) in that (i) decoupling of the on-site interaction does not occur, and (ii) the "mean fields" are operators, not numbers. We also showed that near $T_{c}$ and at low temperatures the resulting thermodynamic behavior has the well-known mean-field form. These results shed new light on the properties of itinerant quantum spin systems and their mean-field description.

We gratefully acknowledge discussions with Dr. W. Metzner and Dr. J. A. Vergés. This work was supported in part by Sonderforschungsbereich No. 341 of the Deutsche Forschungsgemeinschaft.

\footnotetext{
(a) Address after 1 October 1990: Physics Department, MIT, Cambridge, MA 02139.

${ }^{1}$ R. Brout, Phys. Rev. 118, 1009 (1960); C. J. Thompson, Commun. Math. Phys. 36, 255 (1974).

${ }^{2}$ P. A. Pearce and C. J. Thompson, Commun. Math. Phys. 58, 131 (1978); T. Kennedy, E. H. Lieb, and B. S. Shastry, Phys. Rev. Lett. 61, 2582 (1988); J. Stat. Phys. 53, 1019 (1988).

${ }^{3}$ M. C. Gutzwiller, Phys. Rev. Lett. 10, 59 (1963); J. Hubbard, Proc. Roy. Soc. London A 276, 238 (1963); J. Kanamori, Prog. Theor. Phys. 30, 275 (1963).

${ }^{4}$ J. Hubbard, Proc. Roy. Soc. London A 281, 401 (1964); M. C. Gutzwiller, Phys. Rev. 137, A1726 (1965).
}

${ }^{5}$ L. M. Falicov and J. C. Kimball, Phys. Rev. Lett. 22, 997
(1969); in the rare-earth community the model proposed therein is referred to as the "Falicov-Kimball model."

${ }^{6}$ T. Kennedy and E. H. Lieb, Physica (Amsterdam) 138A, 320 (1986); E. H. Lieb, Physica (Amsterdam) 140A, 240 (1986)

${ }^{7}$ U. Brandt and R. Schmidt, Z. Phys. B 63, 45 (1986); 67, 43 (1987)

${ }^{8}$ W. Metzner and D. Vollhardt, Phys. Rev. Lett. 62, 324 (1989).

${ }^{9}$ E. Müller-Hartmann, Z. Phys. B 74, 507 (1989); 76, 211 (1989).

${ }^{10}$ H. Schweitzer and G. Czycholl, Solid State Commun. 69, 171 (1989); 74, 735 (1990).

${ }^{11}$ U. Brandt and C. Mielsch, Z. Phys. B 75, 365 (1989); 79, 295 (1990).

${ }^{12}$ For preliminary reviews, see D. Vollhardt, Int. J. Mod. Phys. B 3, 2189 (1989); E. Müller-Hartmann, Int. J. Mod. Phys. B 3, 2169 (1989).

${ }^{13}$ For a general proof, see P. Lizana, R. Strack, and D. Vollhardt (to be published).

${ }^{14}$ P. G. J. van Dongen (to be published).

${ }^{15}$ The procedure followed here is also of interest for a fundamental reason. Strictly speaking it has never been rigorously proved (Ref. 6) that the low-temperature phase has $A B$ structure for all $T<T_{c}$. Assuming that this is the case, we find that the resulting expression for $T_{c}$ is identical to the value predicted in Ref. 11 when applied to the Bethe lattice. This effectively proves that our assumption is correct.

${ }^{16}$ E. N. Economou, Green's Functions in Quantum Physics (Springer-Verlag, Berlin, 1979).

${ }^{17}$ P. G. J. van Dongen, J. A. Vergés, and D. Vollhardt (to be published).

${ }^{18}$ Note that this finding is not an artifact of the Bethe lattice structure, since the results qualitatively agree with those for a hypercubic lattice in $d=\infty$ (Ref. 11). 